\title{
Predictors affecting durability of epicardial pacemaker leads in pediatric patients
}

\author{
Hiroomi Murayama, MD, ${ }^{a}$ Masanobu Maeda, MD, ${ }^{a}$ Hajime Sakurai, MD, ${ }^{a}$ Akihiko Usui, MD, ${ }^{\mathrm{b}}$ and Yuichi Ueda, $M D^{\mathrm{b}}$
}

Objectives: Despite pacemaker therapy in children and adolescents favoring an initial epicardial approach, predictors of lead failure have not been well clarified. The aim of this study was to assess the long-term outcomes and to determine predictors affecting lead durability in pediatric pacing therapy.

Methods: We reviewed the outcomes of 109 consecutive pacing leads implanted in 55 patients (median age, 5.2 years; range, 31 days -15.8 years), including 38 atrial and 71 ventricular leads. They consisted of 58 (53\%) fishhooks, 37 (34\%) screw-in leads, and $14(13 \%)$ steroid-eluting suture-on leads. Seventy (64\%) were implanted in patients with structural heart disease.

Results: The leads were followed for a median of 6.4 years (range, 3 days-22.9 years). Lead failure occurred in 29 leads (27\%; median of 8.4 years after implantation). Exit block or elevation of pacing threshold was the most common cause (n $=18$ ), but failures did not directly cause patient death. The overall 1-, 5-, 10-, and 15 -year lead survivals were $100 \%, 89.0 \%, 72.5 \%$, and $55.5 \%$, respectively. Multivariate Cox analysis revealed concurrent structural heart disease (relative risk, 2.85; $95 \%$ confidence interval, $1.27-6.42 ; P=.011)$ to be the only significant predictor of lead failure.

Conclusions: Epicardial leads provide a reliable technique for managing rhythmic disturbance problems in the pediatric population. The only significant predictor of lead failure is the presence of structural heart disease.

From the Department of Cardiovascular Surgery, ${ }^{\text {a }}$ Social Insurance Chukyo Hospital, and the Department of Cardio-Thoracic

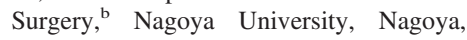
Japan.

Received for publication July 23, 2007; revisions received Sept 6, 2007; accepted for publication Sept 13, 2007.

Address for reprints: Hiroomi Murayama, MD, the Department of Thoracic and Cardiovascular Surgery, Toyohashi Municipal Hospital, Toyohashi, Japan (E-mail: romiwo @ mcn.ne.jp).

J Thorac Cardiovasc Surg 2008;135:361-6

$0022-5223 / \$ 34.00$

Copyright $(\odot) 2008$ by The American Association for Thoracic Surgery

doi:10.1016/j.jtcvs.2007.09.002
$\mathrm{T}$ The majority of children undergoing pacing therapy require long-term pacing throughout their lives. In addition to the more frequent need for pacemaker generator changes because of high pacing rates and high stimulation outputs, frequent lead failure will lead to significant morbidity and mortality at the time of system replacement or at other times if lead failure is sudden and unexpected. The theoretic electronic durability of pacemaker generators is calculated on the basis of measurements of battery voltage and impedance or can be estimated readily by means of past pacing history. ${ }^{1}$ In contrast, lead survival, either of epicardial or endocardial leads, is not exactly predictable. Especially in a pediatric population, epicardial leads are all the more unpredictable in their durability because of the developing body size of young patients or characteristic childhood behaviors, such as creeping in babyhood and vigorous activity in early childhood. Hence we gathered as much data relevant to lead durability or survival as possible. Few studies have examined predictors affecting lead longevity in pediatric epicardial pacing leads. Here we report our data on lead survival and examine predictors affecting lead durability from more than 20 years' experience in epicardial pacing therapy.

\section{Materials and Methods}

This retrospective study represents our experience in permanent pacemaker therapy with consecutive epicardial leads in pediatric patients (age $<16$ years at lead implantation) at Social Insurance Chukyo Hospital. After approval of the institutional review board, pacing 


\section{Abbreviations and Acronyms}

$\mathrm{S}-\mathrm{CHD}=$ structural congenital cardiac disease

leads were identified by serial number, and the following data were obtained from the pacemaker implantation registry and the database: lead type (fishhook, screw-in, or suture-on lead), pacing chamber (atrium or ventricle), measured values at implantation (pacing threshold, sensing amplitude, impedance, and slew rate), duration of use, and mode of failure. The following characteristics of patients undergoing implantation were also obtained: electrophysiologic indications for permanent pacemaker therapy, age at lead implantation, sex, structural congenital cardiac disease (S$\mathrm{CHD}$ ), prior cardiac operation or operations, and age at reimplantation. The minimum energy threshold was calculated by using the previously derived energy formula as follows:

$$
\operatorname{MET}(\mu \mathrm{J})=\frac{\text { Voltage }(\mathrm{V})^{2} \times \text { Pulse width }(\mathrm{ms}) \times 10^{6}}{\text { Impedance }(\Omega) \times 1000 \mathrm{~ms} / \mathrm{s}}
$$

Lead failures were defined as lead replacement or a discontinuation of pacing for any reason, including lead fracture, exit block, excessively elevated pacing threshold, sensing abnormalities, muscle stimulation, or infection.

One hundred nine epicardial pacing leads in 55 patients were identified from the database. Of these, 30 were male and 25 were female, and the median patient age at lead implantation was 5.2 years (range, 31 days-15.8 years). The indications for permanent pacing therapy included atrioventricular block in 41 (75\%), sinus node dysfunction in $11(20 \%)$, and chronic atrial fibrillation with low ventricular rate in $3(5 \%)$. Thirty-four $(62 \%)$ of 55 patients had underlying S-CHD (Table 1). Among the 41 patients with atrioventricular block, 19 had postoperative surgical block. One patient had no S-CHD but had subsequent myocarditis. Dual-chamber pacing (ventricular pacing with atrial synchronization or atrioventricular synchronized pacing mode [DDD]) was used in $36(65 \%)$

\section{Table 1. Structural congenital heart disease}

\begin{tabular}{lc}
\hline Lesion & No. (\%) \\
\hline Tetralogy of Fallot & $7(21)$ \\
Corrected TGA & $5(15)$ \\
Heterotaxia & $5(15)$ \\
Complete AVSD & $4(12)$ \\
Transposition of great arteries & $3(9)$ \\
Ventricular septal defect & $3(9)$ \\
Atrial septal defect & $2(6)$ \\
Congenital mitral insufficiency & $1(3)$ \\
Double-outlet right ventricle & $1(3)$ \\
Tricuspid atresia & $1(3)$ \\
VSD, CoA & $1(3)$ \\
Isolated hypoplastic right ventricle & $1(3)$ \\
Total & $34(100)$ \\
\hline
\end{tabular}

TGA, Transposition of great arteries; $A V S D$, atrioventricular septal defect; $V S D$, ventricular septal defect; $C o A$, coarctation of the aorta.
Table 2. Lead characteristics

\begin{tabular}{lcccc}
\hline Leads & No. (\%) & Position (A/V) & Type & Steroid \\
\hline Medtronic 4951 & $58(53)$ & $33 / 25$ & Fishhook & No \\
Medtronic 5071 & $27(25)$ & $0 / 27$ & Screw-in & No \\
Medtronic 4965 & $14(13)$ & $5 / 9$ & Suture-on & Yes \\
$\quad$ Capsure & & & & \\
Medtronic 6917 AT & $10(9)$ & $0 / 10$ & Screw-in & No \\
Total & $109(100)$ & $38 / 71$ & & \\
\end{tabular}

$A$, Atrium; $V$, ventricle.

patients. In the remaining 19, we used ventricular demand pacing (VVI) or rate-responsive VVI mode (VVIR).

Three types of epicardial leads were primarily used over the 20-year study period. Of 109 total leads implanted, 58 (53\%) were Medtronic 4951 epicardial fishhook leads (Medtronic Inc, Minneapolis, Minn), 37 (34\%) were Medtronic 6917 or 5071 epicardial screw-in leads, and 14 (13\%) were Medtronic 4965 steroid-eluting epicardial suture-on leads (Table 2). The measured values at the time of lead implantation are presented in Table 3.

The lead measurements are expressed as means \pm standard deviation, and other data are expressed as medians with ranges. Differences between the 2 groups were analyzed by using the Student unpaired $t$ test for means of continuous variables and the $\chi^{2}$ test or Fisher exact probability test for categorical variables. Lead survival was calculated by using the Kaplan-Meier method when the data were stratified by lead type, pacing chamber, presence of concurrent S-CHD, and patient's age at lead implantation. Differences between or among the subgroups were verified by using a log-rank test. Cox regression analysis was used to determine predictors of lead failure. Univariate predictors of events with $P$ values of less than .10 were entered into a multivariate Cox proportional hazards model by using a forward selection algorithm.

\section{Results}

There were 854 lead-years for the study period, with a median follow-up interval of 6.4 years (range, 3 days-22.9 years). Complete follow-up data were obtained for all leads. Five leads were censored because of $3(5 \%)$ early deaths, which occurred at 3, 31, and 79 days after pacemaker implantation. These patients died because of severe heart failure in the context of an S-CHD. Pacemaker or lead dysfunction was not implicated in any of the 3 cases. Another lead was censored for elective lead change to an endocardial system at the time of battery depletion at 13.9

Table 3. Implant measurements

\begin{tabular}{lccr}
\hline Measurements & Atrial & Ventricular & $\boldsymbol{P}$ value \\
\hline $\begin{array}{l}\text { Minimum energy } \\
\quad \text { threshold (microJ) }\end{array}$ & $1.8 \pm 2.9$ & $1.6 \pm 2.3$ & .631 \\
Sensing amplitude $(\mathrm{mV})$ & $4.4 \pm 4.2$ & $12.0 \pm 6.0$ & $<.001$ \\
Impedance $(\Omega)$ & $408 \pm 142$ & $542 \pm 179$ & $<.001$ \\
Slew rate $(\mathrm{ms} / \mathrm{s})$ & $0.85 \pm 0.67$ & $1.38 \pm 0.97$ & .009 \\
\hline
\end{tabular}


Table 4. Failed-lead characteristics

\begin{tabular}{lccccc}
\hline Cause & No. (\%) & Position (A/V) & Type (F/S-in/S-on) & Steroid (yes/no) & S-CHD (yes/no) \\
\hline Exit block & $18(62)$ & $6 / 12$ & $14 / 3 / 1$ & $1 / 17$ & $0 / 6$ \\
Lead fracture & $6(21)$ & $2 / 4$ & $4 / 2 / 0$ & $0 / 2$ & $10 / 8$ \\
Sensing problem & $2(7)$ & $0 / 2$ & $2 / 0 / 0$ & $0 / 2$ & $5 / 1$ \\
Infection & $2(7)$ & $1 / 1$ & $2 / 0 / 0$ & $1 / 0$ & $2 / 0$ \\
Lead dislodgement & $1(3)$ & $0 / 1$ & $0 / 0 / 1$ & $2 / 27$ & $1 / 0$ \\
Total & $29(100)$ & $9 / 20$ & $22 / 5 / 2^{*}$ & $20 / 9$
\end{tabular}

$A$, Atrium; $V$, ventricle; $F$, fishhook; $S$-in, suture-in lead; $S$-on, suture-on lead; $S$-CHD, structural congenital heart disease. ${ }^{*} P<.05$.

years after implantation. Twenty-nine (27\%) of 109 leads had to be replaced or abandoned at a median of 8.4 years (range, 1.4-18.8 years) after implantation, resulting in an incidence of $3.4 \%$ per lead per year of follow-up. Exit block or elevation of stimulation threshold was found in 18 leads, making it the most prevalent cause of lead complication, followed by lead fracture in 6 , sensing problems in 2 , infection in 2, and lead dislodgement in 1 (Table 4). Of the 29 failed leads, 20 were implanted in patients who had structural congenital heart disease, including 11 for surgical block.

Exit block or elevated pacing threshold occurred at a median of 6.2 years (range, 1.4-18.3 years) after implantation, including 6 atrial and 12 ventricular leads. It occurred at an equal incidence in atrial and ventricular leads. Most of them $(17 / 18,94 \%)$ were non-steroid-eluting leads, and 14 $(14 / 18,78 \%)$ were fishhook leads. Only 1 steroid-eluting lead complicated exit block 3.3 years after implantation in a patient with heterotaxia who had undergone repeated surgical interventions. Lead fractures were encountered in 6 leads at a median of 12.1 years (range, $4.2-18.8$ years) after implantation. All fractured leads except 1 (5/6, 83\%) had been implanted in the patients with surgical block through a median sternotomy approach. Sensing problems were incidentally discovered on a routine Holter electrocardiographic recording, including one oversensing and one undersensing. A pair of atrial and ventricular leads implanted in a male patient with atopic dermatitis were infected with methicillin-resistant Staphylococcus aureus. Skin erosion of the superficial pacemaker pocket was first noted 9.0 years after implantation and finally extended to mediastinitis, necessitating a rethoracotomy and removal of the entire pacemaker system. The patient was successfully treated with a new transvenous pacemaker implantation. Lead dislodgement occurred in 1 steroid-eluting lead, which had been implanted by means of suturing on the epicardium.

The overall 1-, 5-, 10-, and 15-year freedoms from lead failure were $100 \%, 89.0 \%, 72.5 \%$, and $55.5 \%$, respectively (Figure 1). No differences were noted among the lead types, where the 5-year freedoms from lead failure were $91.3 \%$ for fishhook, $87.5 \%$ for screw-in, and $84.4 \%$ for steroid eluting suture-on leads, respectively. When the leads were com- pared based on steroid elution, the 5-year freedom from lead failure was $89.9 \%$ for non-steroid-eluting leads, showing no significant difference from the above data for steroid-eluting leads (Figure 2). Neither the pacing chamber nor the patient's age at lead implantation affected lead failure rates. The only significant difference was noted in the lead failure rates among those with concurrent S-CHD. The leads were more likely to survive in patients without S-CHD than in those with S-CHD $(P=.008)$. The freedoms from lead failure at $1,5,10$, and 15 years were, respectively, $100 \%$, $93.5 \%, 79.0 \%$, and $74.6 \%$ in the former group and $100 \%$, $86.2 \%, 66.1 \%$, and $31.4 \%$ in the latter (Figure 3).

Table 5 shows the relative risks of possible predictors for lead failure evaluated by means of univariate Cox regression analysis. In the 11 variables the implanted lead measurements (minimum energy threshold, sensing amplitude, impedance, and slew rate) were examined within each subgroup of atrial and ventricular leads, respectively. Univariate analysis detected S-CHD, sex, and prior cardiac surgery as significant predictors $(P<.05)$ and age at lead implantation as of borderline significance $(P=.077)$. Subsequent

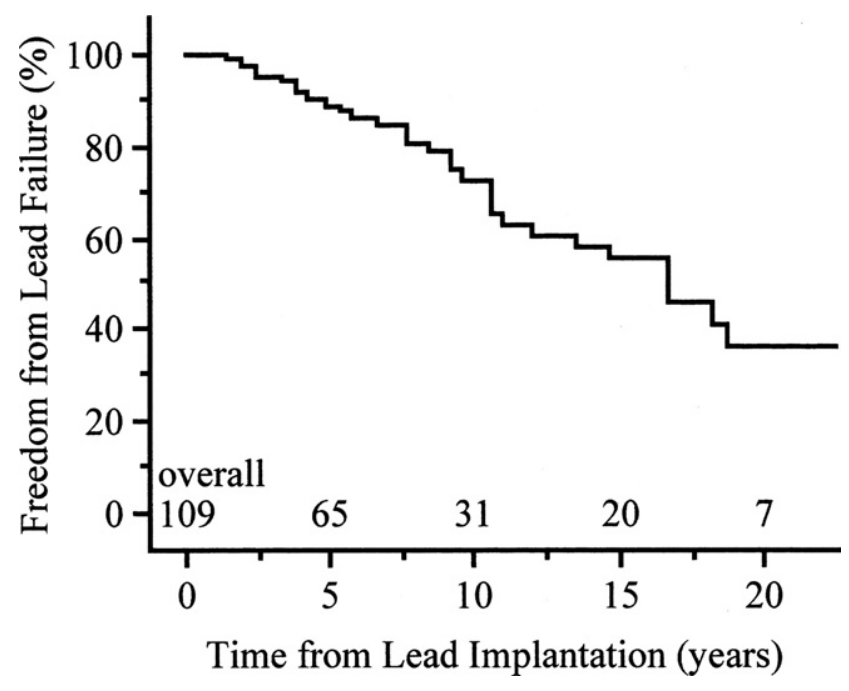

Figure 1. Kaplan-Meier freedom from lead failure in overall epicardial leads. 


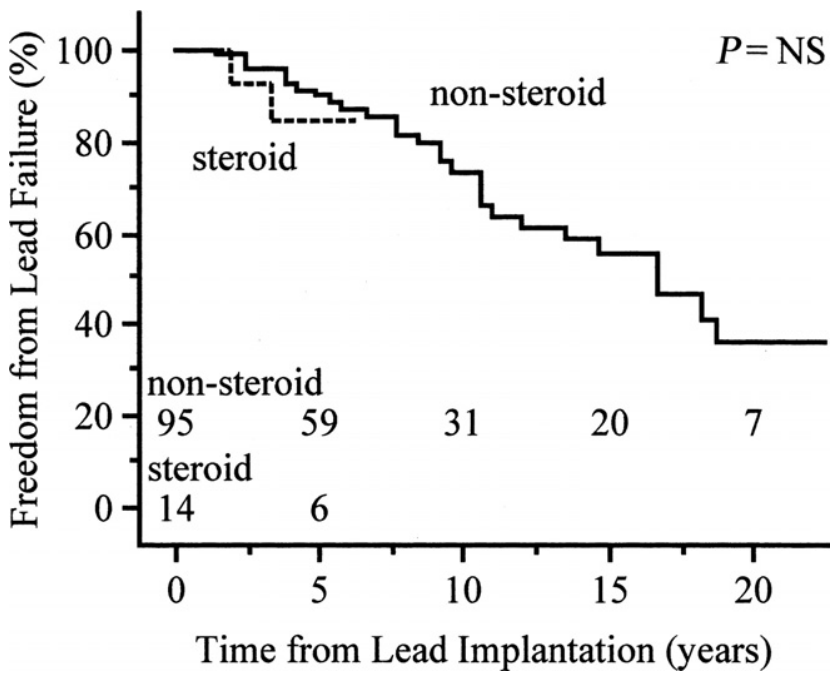

Figure 2. Kaplan-Meier freedom from lead failure for non-steroid-eluting (solid line) versus steroid-eluting (broken line) epicardial leads. NS, Not significant.

multivariate analysis with a stepwise forward Cox proportional hazards model confirming the presence of S-CHD was the only significant predictor of lead failure (relative risk, $2.85 ; 95 \%$ confidence interval, $1.27-6.42 ; P=.011$ ).

\section{Discussion}

Epicardial leads have been reported to have only short-term durability and show a high rate of lead failure. Therefore the endocardial approach has gained favor in general use. ${ }^{2-5}$ Nevertheless, the epicardial approach for pacemaker ther-

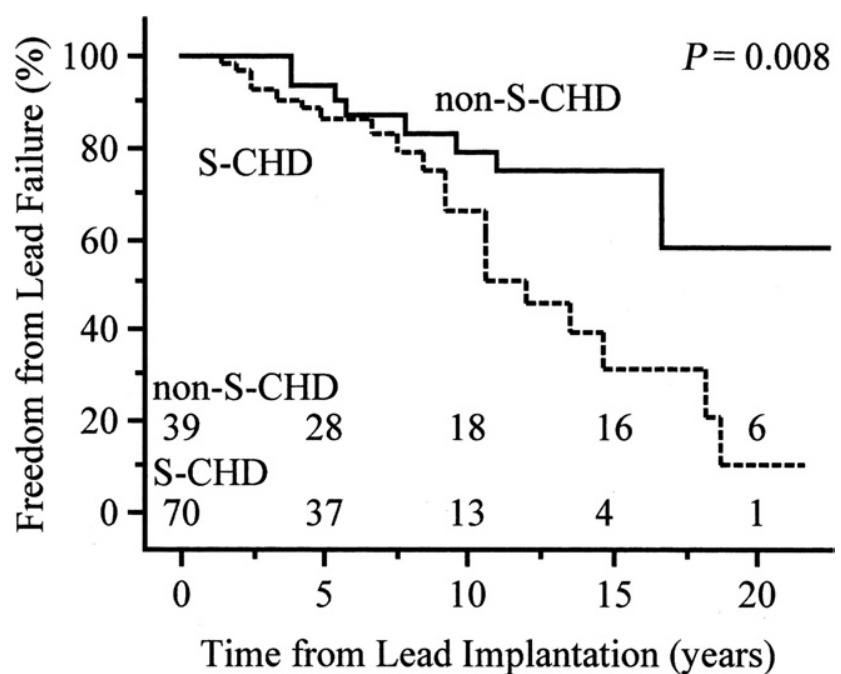

Figure 3. Kaplan-Meier freedom from lead failure in patients with (broken line) or without (solid line) structural congenital heart disease. S-CHD, Structural congenital heart disease.
Table 5. Univariate Cox regression analysis on epicardial lead durability

\begin{tabular}{|c|c|c|c|}
\hline Variables & $\mathbf{R R}$ & $95 \% \mathrm{CI}$ & $\begin{array}{c}P \\
\text { value }\end{array}$ \\
\hline Age at lead implantation (y) & 0.91 & $0.82-1.01$ & .077 \\
\hline Female sex & 0.40 & $0.17-0.94$ & .036 \\
\hline Structural congenital heart disease & 2.85 & $1.27-6.42$ & .011 \\
\hline Prior cardiac surgery & 2.28 & $1.01-5.16$ & .047 \\
\hline Pacing chamber (atrial, 0 ; ventricular, 1 ) & 1.18 & $0.53-2.62$ & .688 \\
\hline \multicolumn{4}{|l|}{ Lead type } \\
\hline Fishhook (vs suture-on) & 0.73 & $0.15-3.42$ & .697 \\
\hline Screw-in (vs suture-on) & 0.31 & $0.05-1.80$ & .191 \\
\hline Steroid elution & 1.66 & $0.35-7.85$ & .519 \\
\hline \multicolumn{4}{|l|}{ Minimum energy threshold } \\
\hline Atrial (microJ) & 0.95 & $0.68-1.32$ & .753 \\
\hline Ventricular (microJ) & 1.07 & $0.92-1.21$ & .423 \\
\hline \multicolumn{4}{|l|}{ Sensing amplitude } \\
\hline Atrial (mV) & 1.08 & $0.97-1.20$ & .173 \\
\hline Ventricular (mV) & 1.06 & $0.99-1.13$ & .105 \\
\hline \multicolumn{4}{|l|}{ Impedance } \\
\hline Atrial $(\Omega)$ & 1.00 & $1.00-1.01$ & .662 \\
\hline Ventricular $(\Omega)$ & 1.00 & $1.00-1.00$ & .115 \\
\hline \multicolumn{4}{|l|}{ Slew rate } \\
\hline Atrial (ms/s) & 0.83 & $0.15-4.65$ & .828 \\
\hline Ventricular (ms/s) & 1.35 & $0.81-2.25$ & .255 \\
\hline
\end{tabular}

$R R$, Relative risk; $\mathrm{Cl}$, confidence interval.

apy continues to be required. Some children and young adults evidence relative or absolute contraindications to the transvenous approach because of small body size, presence of a right-to-left shunt, or absence of venous access caused by congenital anomalies or surgical interventions. ${ }^{6}$ Other children might be able to benefit from either an endocardial or epicardial system. Endocardial leads, however, might complicate venous thrombosis, vascular obstruction, or atrioventricular valve integrity. ${ }^{7-11}$ There are potential concerns about disproportion of lead length caused by somatic growth or difficulty in cases of lead extraction. ${ }^{12-14}$ Because the majority of children undergoing pacing therapy will require pacing for life, they are commonly exposed to such risks for a nearly normal lifespan. Therefore, although it is technically feasible to implant endocardial leads in children, we apply the epicardial approach. Our policy in practicing permanent pacemaker therapy in a pediatric population has been that epicardial pacing takes precedence in principle for all patients weighing less than $30 \mathrm{~kg}$ or those 16 years of age or younger.

We found that lead failures occurred in 29 leads, $27 \%$ of all implanted epicardial leads during a median of 6.4 years, and the lead failure rate determined by means of KaplanMeier analysis was found to be $89.0 \%$ and $72.5 \%$ at 5 and 10 years, respectively. These observations are consistent with those of previous pediatric epicardial lead studies. 
Cohen and colleagues ${ }^{15}$ reported the incidence of epicardial lead failure observed in $16 \%$ of leads for a median follow-up interval of 2.4 years, whereas Thomson and associates ${ }^{16}$ reported it in $34 \%$ for a median of 2.3 years. In such studies various probabilities of lead survival rate were reported in a variety of cohorts, ranging from 58\% to $91 \%$ at 5 years and $30 \%$ to $72 \%$ at 10 years. ${ }^{12,15-18}$ Walker and coworkers ${ }^{19}$ reported an excellent outcome of epicardial leads implanted in an adult population with S-CHD, showing $92 \%$ survival at 5 years and $84 \%$ at 10 years. This wide spectrum of lead survival rates among the study groups highlights the fact that the epicardial cardiac pacing is complex and that the performance of the leads might not be the sole reason for their high failure rate in the context of complicated patient characteristics. The survival rate in adults suggests that epicardial leads might have superior potential for their durability in children as well.

In this study the presence of concurrent S-CHD was identified as predictive of subsequent lead failure. The Kaplan-Meier analysis showed that the leads implanted in patients with S-CHD had a significantly higher failure rate than that of those implanted in patients without S-CHD. In multivariate Cox proportional hazards analysis, S-CHD was revealed to be the only significant predictor affecting lead survival. The leads implanted in the patients with S-CHD had 2.9 times higher risk for lead failure compared with those implanted in patients without S-CHD. Cohen and colleagues ${ }^{15}$ reported no relationship between lead durability and S-CHD, whereas in the same study they mentioned the possibility that S-CHD and cardiac surgery affect longterm pacing thresholds and that increased pacing thresholds are associated with reduced lead longevity.,3

Scar tissue, fat, fibrosis, or myocardial degeneration can affect the pacing threshold. We speculate that there might be a number of unquantifiable factors that could increase the risks of lead failure in the epicardium and myocardium, where the leads were implanted in such patients with S-CHD.

We, however, have dismissed steroid elution, lead position, patient age at lead implantation, or measurement values of implanted leads as predictors for lead complications. Many recent reports have mentioned the enhanced effect of steroid-eluting epicardial leads. Beaufort-Krol and cowork$\mathrm{ers}^{20}$ reported their performance, including longevity, to be very similar to those of conventional endocardial leads. Thomson and associates ${ }^{16}$ found a $76 \%$ survival of steroideluting epicardial leads at 5 years, and Cohen and colleagues ${ }^{15}$ reported an $83 \%$ survival at 5 years. Beder and coworkers $^{21}$ reported an opposite hazard that both early and late precipitous lead failures were still occurring in steroideluting leads, despite their superior electrical performances. In this report we have a rather small number of steroideluting leads with a relatively short observation period, and they were not shown to improve lead survival. Their use obviously means a lower pacing threshold and less drain on batteries and thus longer battery life, not to mention less subsequent surgical intervention. ${ }^{22}$

The primary goal of permanent pacemaker therapy is stable pacing with minimum patient risk and avoidance of complications for their lifespan. The present study of epicardial leads over a 20-year period among Japanese patients confirmed that satisfactory lead durability could be achieved through careful observation and meticulous management. Our experience, as well as the excellent results of Walker and coworkers, ${ }^{19}$ encourages us to keep offering epicardial pacing to particular patients. We should consider the feasibility not only of early performance but also of how best to achieve a lifetime of successful, uninterrupted pacing. We believe that our experiences described here might be helpful in the understanding of how to pace this population.

This is a retrospective analysis over a long observation period. Choice of lead has varied among surgeons, with technical development over time. The relatively small number of leads limits statistical power, and further accumulation of experiences is required.

We thank Nobuyuki Hamajima, MD, MPH, PhD, from the Department of Preventive Medicine/Biostatistics and Medical Decision Making, Nagoya University Graduate School of Medicine, for his kind statistical review. We also thank Masami Nagashima, MD, and Masaki Matsushima, MD, from the Department of Pediatric Cardiology for patient management and follow-up.

\section{References}

1. Mallela VS, Ilankumaran V, Rao NS. Trends in cardiac pacemaker batteries. Indian Pacing Electrophysiol J. 2004;4:201-12.

2. Williams WG, Izukawa T, Olley PM, Trusler GA, Rowe RD. Permanent cardiac pacing in infants and children. Pacing Clin Electrophysiol. 1978;1:439-47.

3. Sachweh JS, Vazquez-Jimenez JF, Schöndube FA, Daebritz SH, Dörge H, Mühler EG, et al. Twenty years experience with pediatric pacing: epicardial and transvenous stimulation. Eur J Cardiothorac Surg. 2000;17:455-61.

4. Till JA, Jones S, Rowland E, Shinebourne EA, Ward DE. Endocardial pacing in infants and children $15 \mathrm{~kg}$ or less in weight: medium-term follow-up. Pacing Clin Electrophysiol. 1990;13:1385-92.

5. Ayabakan C, Rosenthal E. Endocardial pacemaker implantation in neonates and infants. Indian Pacing Electrophysiol J. 2006;6:57-62.

6. Gillette PC, Shannon C, Blair H, Garson A Jr, Porter CJ, McNamara DG. Transvenous pacing in pediatric patients. Am Heart J. 1983;105: 843-7.

7. Korkeila PJ, Saraste MK, Nyman KM, Koistinen J, Lund J, Airaksinen KEJ. Transesophageal echocardiography in the diagnosis of thrombosis associated with permanent transvenous pacemaker electrodes. Pacing Clin Electrophysiol. 2006;29:1245-50.

8. Figa FH, McCrindle BW, Bigras JL, Hamilton RM, Gow RM. Risk factors for venous obstruction in children with transvenous pacing leads. Pacing Clin Electrophysiol. 1997;20:1902-9.

9. Ruge H, Wildhirt SM, Poerner M, Mayr N, Bauernshmitt R, Martinoff $\mathrm{S}$, et al. Severe superior vena cava syndrome after transvenous pacemaker implantation. Ann Thorac Surg. 2006;82:e41-2.

10. Riezebos RK, Schroeder-Tanka J, de Voogt WG. Occlusion of the proximal subclavian vein complicating pacemaker lead implantation. Europace. 2006;8:42-3. 
11. Lin G, Nishimura RA, Connolly HM, Dearani JA, Sundt TM III, Hayes DL. Severe symptomatic tricuspid valve regurgitation due to permanent pacemaker or implantable cardioverter-defibrillator leads. J Am Coll Cardiol. 2005;45:1672-5.

12. Silvetti MS, Drago F, Grutter G, De Santis A, Di Ciommo V, Rava L. Twenty years of paediatric cardiac pacing: 515 pacemakers and 480 leads implanted in 292 patients. Europace. 2006;8:530-6.

13. Madigan NP, Curtis JJ, Sanfelippo JF, Murphy TJ. Difficulty of extraction of chronically implanted tined ventricular endocardial leads. J Am Coll Cardiol. 1984;3:724-31.

14. Myers MR, Parsonnet V, Bernstein AD. Extraction of implanted transvenous pacing leads: a review of a persistent clinical problem. Am Heart J. 1991;121:881-8.

15. Cohen MI, Bush DM, Vetter VL, Tanel RE, Wieand TS, Gaynor JW, et al. Permanent epicardial pacing in pediatric patients: seventeen years of experience and 1200 outpatient visits. Circulation. 2001;103: 2585-90.

16. Thomson JDR, Blackburn ME, Van Doorn C, Nicholls A, Watterson KG. Pacing activity, patient and lead survival over 20 years of permanent epicardial pacing in children. Ann Thorac Surg. 2004;77:1366-70.

17. DeLeon SY, Ilbawi MN, Backer CL, Idriss FS, Paul MH, Zales VR, et al. Exit block in pediatric cardiac pacing: comparison of the suture- type and fishhook epicardial electrodes. J Thorac Cardiovasc Surg. 1990;99:905-10.

18. Cohen MI, Vetter VL, Wemovsky G, Bush DM, Gaynor JW, Iyer VR, et al. Epicardial pacemaker implantation and follow-up in patients with a single ventricle after the Fontan operation. J Thorac Cardiovasc Surg. 2001;121:804-11.

19. Walker F, Siu SC, Woods S, Cameron DA, Webb GD, Harris L. Long-term outcomes of cardiac pacing in adults with congenital heart disease. J Am Coll Cardiol. 2004;43:1894-901.

20. Beaufort-Krol GCM, Mulder H, Nagelkerke D, Waterbolok TW, BinkBoelkens MTE. Comparison of longevity, pacing, and sensing characteristics of steroid-eluting epicardial versus conventional endocardial pacing leads in children. J Thorac Cardiovasc Surg. 1999;117: 523-8.

21. Beder SD, Kuehl KS, Hopkins RA, Tonder LM, Mans DR. Precipitous exit block with epicardial steroid-eluting leads. Pacing Clin Electrophysiol. 1997;20:2954-7.

22. Horenstein MS, Hakimi M, Walters H III, Karpawich PP Chronic performance of steroid-eluting epicardial leads in a growing pediatric population. Pacing Clin Electrophysiol. 2003;26: 1467-71.

\title{
Interactive eLearning Activities http://learning.ctsnet.org
}

The Joint Council on Thoracic Surgery Education was pleased to introduce a series of unique eLearning activities to CTSNet users at the AATS annual meeting in Philadelphia. Sponsored by several cardiothoracic surgical groups, this exciting new educational tool contains narrated videos of actual surgical procedures followed by a series of questions and an evaluation to earn Continuing Medical Education credit.

* Diagnosis and Management of Complications of Mitral Valve Repair

* Off Pump Coronary Artery Bypass

* OPCAB Debriefing

* Pediatric Cardiopulmonary Bypass Emergency Situations

\section{Experience these new educational tools today and provide us with your feedback. Go to: http://learning.ctsnet.org}

William E. Baumgartner, Chair

Joint Council on Thoracic Surgery Education

\author{
Sponsored By: \\ American Association for Thoracic Surgery \\ The Society of Thoracic Surgeons \\ European Association for Cardio-Thoracic Surgery \\ Children's Memorial Hospital
}

